AGENS online

\section{Angebote zur Vernetzung der Arbeitsgruppe}

Während der letzten AGENS-Treffen wurde immer wieder der Wunsch geäußert und diskutiert, dass eine Art Methodensammlung für die Arbeit mit Sekundärdaten entsteht, auf die jede:r Forschende zurückgreifen kann. Auch die Etablierung einer Austauschplattform abseits der Treffen, bspw. über ein Online-Forum, wurde angeregt.

Konkrete Wünsche für eine bessere Vernetzung untereinander wurden über eine Online-Umfrage im März 2020 bei den rund 500 AGENS-Mitgliedern erhoben. Die Ergebnisse sollten Möglichkeiten für die Förderung des wissenschaftlichen Austausches aufzeigen. Zur Beteiligung wurde über den AGENS-Mailverteiler und den AGENSTwitteraccount @agens_de aufgerufen.

Insgesamt nahmen 65 AGENS-Mitglieder und über Twitter erreichte Interessierte an der Umfrage teil. 28 von ihnen gaben an, dass für sekundärdatenbezogene publikationswürdige Inhalte (insbesondere Methoden und Auswertungsskripte) bisher kein geeignetes Medium gebe und ein Sekundärdatenjournal diese Lücke schließen könne (24 von 28). Jeweils 7 Teilnehmende nannten ergänzend einen Blog oder eine strukturierte PDF-Sammlung als Möglichkeit. Zwei Drittel aller Teilnehmenden $(n=41)$ halten eine eigenständige Webseite der AGENS als sinnvoll für die Kommunikation in der Arbeitsgruppe, etwas mehr als die Hälfte $(n=35)$ zudem ein Forum für den fachlichen Austausch. Alle Ergebnisse zur Online-Umfrage können gerne per Mail bei uns angefordert werden.

Die Präsenz der AGENS auf ResearchGate und Twitter war der erste Schritt zur stärkeren Nutzung digitaler Kommunikationskanäle. Seit September 2020 sind auch die Webseite (https://www.agens.group) und das Forum (https://forum.agens.group) der AGENS online. Die Server dafür stellt freundlicherweise die PMV forschungsgruppe zur Verfügung. Die Webseite macht die vielseitigen Aktivitäten der AGENS transparent: auf alle bisherigen Publikationen kann zentral zugegriffen werden, aktuelle Informationen zu AGENSSchools und den MethodenWorkshops sowie AG Treffen und durch die AG ausgerichtete Sessions auf Kongressen werden bereitgestellt. Auf dem Forum mit seiner stetig wachsenden Nutzerzahl werden erste gemeinsame Projekte besprochen. Über Twitter werden mittlerweile 280 Interessierte unkompliziert mit neuesten Informationen aus dem Bereich der Sekundärdatenanalyse versorgt.

Der Start der AGENS in die Onlinewelt war erfolgreich! Gleichzeitig leben diese Angebote von aktiven Forscher:innen, die das Onlineangebot ausweiten und mit Leben und Content füllen. Der Zugang ist einfach: schauen Sie im Forum in der Gruppe „AGENS online“ vorbei, treten Sie direkt mit uns in Kontakt und vor allen Dingen, nutzen und bewerben Sie aktiv die AGENS Website und das Forum, unsere ResearchGate Gruppe sowie unseren Twitter-Account. Konkrete Themen, die uns aktuell bewegen sind die Etablierung einer Methoden- und Skriptsammlung und die Frage, ob das Forum zukünftig auf einer Plattform wie Reddit oder Discord geführt werden sollte.

Christoph Stallmann, Institut für

Sozialmedizin und

Gesundheitssystemforschung, Med.

Fakultät, Otto-von-Guericke-Universität Magdeburg, Magdeburg

Ingo Meyer, PMV forschungsgruppe, Köln 